\title{
Single Layer Coating Photonic Crystal Fiber Biosensor based on Surface Plasmon Resonance
}

\author{
P Vinod Kumar, Snivash, Mohammed Fahimullah A, K Vinay Gokul
}

\begin{abstract}
This paper is a demonstration for the design of photonic crystal fiber biosensor which is based on the phenomenon of surface plasmon resonance. The plasmonic coating covers the outer layer of the Photonic Crystal fiber to ease the fabrication process, we choose Gold. To quantitatively measure the represented design, we utilize a technique called as the Finite element method (FEM). To accomplish the sensitivities of 4300nm/RIU and 408.468 RIU ${ }^{-1}$ we apply the methodologies of wavelength and amplitude interrogation models. This design yielded a resolution of $2.33 \times 10^{-5}$ respectively. A fluctuation from 1.33 to 1.39 in the analyte refractive index can be identified and measured by this design. The sensing range of the design is wide and may also be used in biological detection.
\end{abstract}

Keywords: Biosensor, Resolution, PCF, SPR

\section{INTRODUCTION}

In recent trends, there's a keen interest for the study of Surface plasmon resonance effect based Photonic Crystal Fiber because of its good properties and its advantages. SPR based PCF has been used for physical, biological, chemical applications. It is very important to choose the proper design and plasmonic material. The sensor performance is dependent on the proper selection of plasmonic material. Several kinds ofplasmonic material have been used. The most common type ofplasmonic material is being used is Gold because of itsproperties which includes chemically stability, oxidization and it exhibits high sensitivity because of the large resonancepeaks when compared with other plasmonic metals [7].WhenSPR based PCF are compared with fiber-based sensors, theformer provides more sensitivity and resolution whencompared with the latter. Several designs have been proposedby researches in the past few years for different applications.Md Hasan [3] proposed an extremely sensitive PCF basedon Surface Plasmon Resonance which showed wavelengthsensitivity up to $2200 \mathrm{~nm} / \mathrm{RIU} 1.33$ to 1.36 . Azzam [1]proposed a biosensor based on SPR whose sensitivity was $2400 \mathrm{~nm} R \mathrm{RI}^{-1}$ in visible or near IR region. A square array PCF based SPR refractive index was proposed by Liu [8] had a remarkable sensitivity up to $7250 \mathrm{~nm} / \mathrm{RIU}^{-1}$ in the range of $1.38-1.42$ and it had a

Revised Version Manuscript Received on Jun 20, 2019.

P Vinod Kumar, Electronics and Communication Engineering, SRM Institute of Science and Technology, Chennai, India.

S Nivash, Electronics and Communication Engineering, SRM Institute of Science and Technology, Chennai, India.

Mohammed Fahimullah A, Electronics and Communication Engineering, SRM Institute of Science and Technology, Chennai, India.

K Vinay Gokul, Electronics and Communication Engineering, SRM Institute of Science and Technology, Chennai, India. good resolution of $138 \times 10^{-5} . \mathrm{Li}$ [5] proposed a multi-coating PCF with SPR at near wavelength for detecting higher RI. In this paper the plasmonic material has been coated internally which is a tedious process in real time. So, for ease fabrication gold layer has been coated outside the PCF in our paper. WeiquanSu[6] proposed a D-shaped symmetrical dual PCF which showed a high resolutionup to $6.82 \times 10^{-6}$ in the range of $1.36-1.41$. The importantmeasures of the sensor performance are to make the fabricationeasy and comfortable. The proposed PCF biosensor design hasa simple structure and is easier to fabricate using the stack anddraw [4] process.

\section{THEORETICAL MODELLING}

Fig.1 represents the two-dimensionalillustration of the preferred single layer PCF biosensor. Thispresented design was designed using COMSOL Multiphysics.Fiber design is characterized by its parameters $\operatorname{pitch}(\Lambda)$ anddiameter $\left(d_{1}\right.$, $\mathrm{d}_{2}$ ). The $\Lambda$ denotes the measure between the coreof air holes, also $d_{1}$ and $d_{2}$ are the diameters of the smallerand bigger circles respectively. The value for these parameters is $\Lambda=$ $2 \mu \mathrm{m}, \mathrm{d}_{1}=0.25 \times \Lambda$, and $\mathrm{d}_{2}=0.5 \times \Lambda$. A small hole in thecore of the fiber is introduced, and its diameter is given asd $_{c}=0.15 \times$ $\Lambda$ and the thickness of the plasmonic material ist $\mathrm{au}_{\mathrm{au}}=30 \mathrm{~nm}$. The thickness of the analyte region on top of thegold layer is $\mathrm{t}_{\text {Anal }}=0.8 \mu \mathrm{m}$. Perfectly Matched Layer is theexternal layer of the entire structure is used to absorb the electromagnetic radiations.

The number of degrees of freedom of a computed mesh generation is 102587 and the number of elements is 19938. The photonic crystal fiber is made up of Silica.The refractive index of the fiber which is made using Silica can be calculated using Sellmeier equation [3] is:

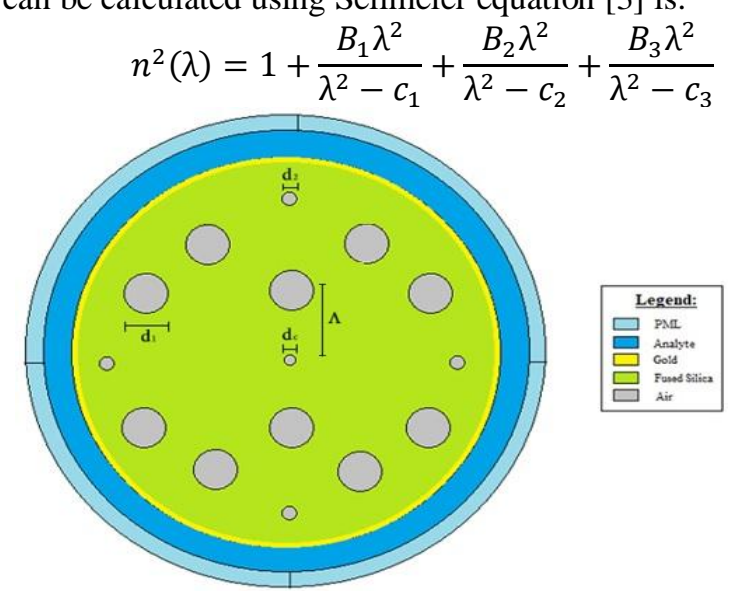

Fig. 1. The cross-sectional view of the proposed PCF sensor whose parameters are $\Lambda=2 \mu \mathrm{m}$, $\mathrm{d} 1=0.25 \times \Lambda$ and $\mathrm{d} 2=0.5 \times \Lambda$ and $\mathrm{dc}=0.3 \times \Lambda$.

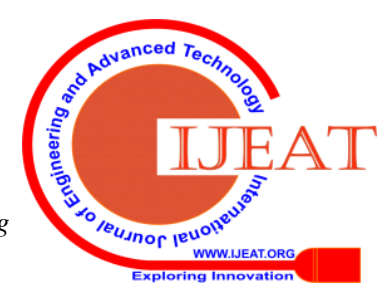


where $\mathrm{n}$ is RI of silica at wavelength $l$ in microns. $\mathrm{B}_{1}, \mathrm{~B}_{2}, \mathrm{~B}_{3}$ and $c_{1}, c_{2}, c_{3}$ are Sellmeier coefficients whose values are given by $0.696166300,0.407942600,0.897479400$ and $4.67914826103 \mu m^{2}, 1.35120631102 \mu m^{2}, 97.9340025 \mu m^{2}$ respectively. The permittivity of gold is obtained using dielectric function of gold using Drude-Lorentz [3] given as:

$$
\epsilon_{A u}=\epsilon_{\infty}-\frac{\omega_{D}^{2}}{\omega\left(\omega+j \gamma_{D}\right)}-\frac{\Delta \Omega_{L}^{2}}{\left(\omega^{2}-\Omega_{L}^{2}\right)+j \Gamma_{L} \omega}
$$

where the permittivity $(\varepsilon \infty)$ of gold at high frequencies is 5.9673. $\omega_{\mathrm{D}}, \gamma_{D}, \omega, \Omega_{L}$ parameters are explained in [7].

\section{SIMULATION RESULTS}

The parameters of the Photonic Crystal Fiber determines the Sensor performance. The evanescent field getsoriginated from the core and when this field hits the gold (plasmonic metal) surface plasmon waves are produced in the metal-dielectric interface. There is a particular wavelength called resonant wavelength at which the highest energy from fundamental mode gets transferred to the plasmonic mode and hence a sharp peak in the loss curve can be observed as shownin Fig.3.
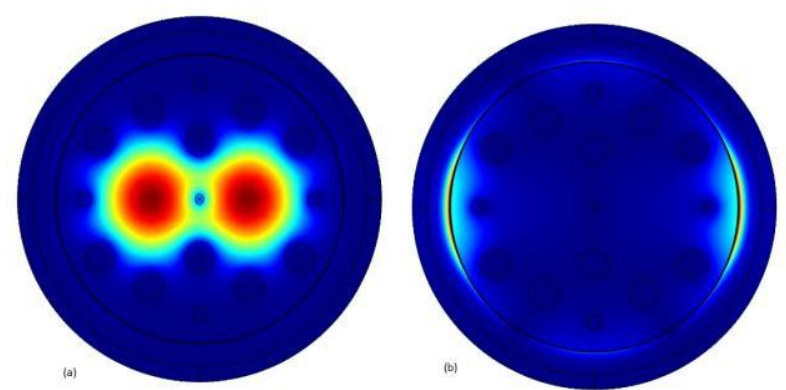

Fig. 2. Mode field distribution at (a)Fundamental mode and (b)Plasmonic mode for analyte $\mathrm{RI}=1.36$ and the parameters of PCF include $\Lambda=2 \mu \mathrm{m}, \mathrm{d} 1=0.25 \times \Lambda, \mathrm{d} 2=0.5 \times \Lambda$ , $\mathrm{dc}=0.3 \times \Lambda$ and $\mathrm{t}_{\mathrm{au}}=30 \mathrm{~nm}$.

By using the change in the loss peak the refractive index of an analyte can be determined. $\mathrm{X}$-polarization loss is very less when compared to Y-polarization loss, only Y-polarization was considered throughout the simulation. Fig.2(a) represents the Fundamental mode Electric field distribution for analyte refractive index 1.36 and 2(b) represents the distribution of the electric field of fundamental mode for analyte RI index 1.36. From Fig[2a] and Fig.[2b] it can be understood that the electric field which has been originated from the core in Fig[2a] reaches the plasmonic material in Fig[2b].

The confinement loss which is expressed in $\mathrm{dB} / \mathrm{cm}$ can be calculated using [9]:

$$
\alpha(d B / c m)=8.686 \times k_{0} \times \operatorname{Im}\left(n_{e f f}\right) \times 10^{4}
$$

where $\mathrm{k}_{0}=2 \Pi / \lambda$ represents the free space propagation constant, $\lambda$ represents the wavelength which is expressed in $\mu \mathrm{m}$ and $\operatorname{Im}\left(n_{\text {eff }}\right)$ represents effective refractive index imaginary $\operatorname{part}\left(\mathrm{n}_{e f f}\right)$.

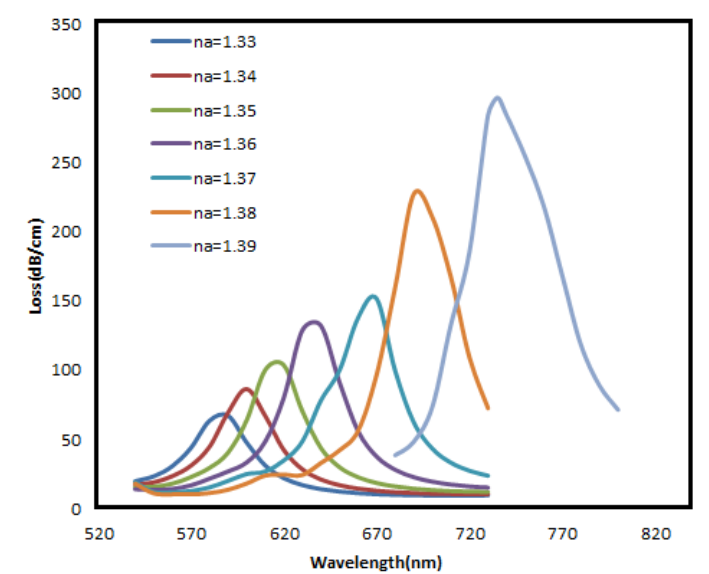

Fig. 3. Fundamental loss curve for analyte RI fluctuating from 1.33 to 1.39 . The PCF parameters include $\Lambda=2 \mu \mathrm{m}$, $\mathrm{d} 1=0.25 \times \Lambda, \mathrm{d} 2=0.5 \times \Lambda, \mathrm{dc}=0.3 \times \Lambda$ and $\mathrm{t}_{\mathrm{au}}=30 \mathrm{~nm}$.

There is always a shift in the loss depth whenever the RI of the analyte is changed as shown in Fig.3. When the analyte refractive index is increased it leads to redshift of the curve therefore the resonant wavelength also gets redshifted. When the RI of the analyte is decreased blueshift occurs, therefore the resonant wavelength also gets blue shifted. It can be understood that the shift in the loss depth is due to the change in the analyte Refractive index. The effective refractive index of the plasmonic mode is dependent on change of analyte refractive index. The resonant Wavelength for analyte RI of $1.33,1.34,1.35,1.36,1.37,1.38,1.39$ is 590nm, 600nm, 620nm, 640nm, 670nm ,692nm,735nm and itscorresponding losses are $66.785 \mathrm{~dB} / \mathrm{cm}$, $86.049554 \mathrm{~dB} / \mathrm{cm}, 103.4302 \mathrm{~dB} / \mathrm{cm}$,

$13.0524 \mathrm{~dB} / \mathrm{cm}, 151.384 \mathrm{~dB} / \mathrm{cm}, 228.335 \mathrm{~dB} / \mathrm{cm}, 29.8218 \mathrm{~dB} / \mathrm{c}$ $\mathrm{m}$. Whenever there is achange in the RI of the analyte, resonant wavelength alwaysgets shifted either towards the higher wavelength or towardsthe lower wavelength.The sensor's Sensitivity based onwavelength methodology is dependent on the resonantwavelength shift.

The wavelength Sensitivity $S_{\lambda}$ which is expressed in $\mathrm{nm} / \mathrm{RIU}$ can be calculated using,

$$
S_{\lambda}(n m / R I U)=\Delta \lambda_{\text {peak }} / \Delta n_{a}
$$

where $\Delta \mathrm{n}_{\mathrm{a}}$ represents the analyte RI changes and $\Delta \lambda_{\text {peak }}$ represents the difference of the successive resonant peaks. Theresonant wavelength shift of 10, 20, 20, 30, 22, 43 for analyteRI value carried in the range of 1.33 to 1.39 with an incrementof 0.01. By using equation the wavelength sensitivitiesare1000, 2000, 2000, 3000, 2200, 4300nm/RIU respectively. Theaverage wavelength sensitivity for analyte RI range 1.33-1.39is $2616 \mathrm{~nm} / \mathrm{RIU}$.To indicate the performance of the sensor, there isa parameter called Resolution can be calculated which isexpressed in RIU using [2]:

$$
R(R I U)=\Delta n_{a} \times \Delta \lambda_{\text {min }} / \Delta \lambda_{\text {peak }}
$$

Where $\Delta \lambda_{\text {peak }}$ and $\Delta \lambda_{\text {min }}$ represent the difference of the successive resonant wavelength and the minimum spectral resolution. Assume $\Delta \lambda_{\min }$ 
$=0.1 \mathrm{~nm}, \quad \Delta \mathrm{n}_{\mathrm{a}}, \quad=$
calculated $\Delta \lambda_{\text {peak }}=43 \mathrm{~nm}$.

0.01

and

the

The highest Resolution recorded for our design is $2.33 \times 10^{-5}$. So, this sensor can detect refractive index changes up to 0.00001 . There is also another important parameter called Amplitude Sensitivity to measure the sensor performance. The Amplitude sensitivity $S_{A}$ has been analyzedand it can be calculated which is expressed in $\mathrm{RIU}^{-1}$ using,

$$
S_{A}\left(R I U^{-1}\right)=-\frac{1}{\alpha\left(\lambda, n_{a}\right)} \frac{\delta\left(\lambda, n_{a}\right)}{\delta n_{a}}
$$

Where $\delta \alpha\left(\lambda, n_{a}\right)$ refers to the confinement loss difference of two successive analyte RI and $\alpha\left(\lambda, n_{a}\right)$ is the confinement loss. For all RI change of analyte fluctuating from 1.33 to 1.39 whose RI change is in the order of 0.01 , their amplitude sensitivities have been calculated and from the measured values it has been found that the maximum amplitude sensitivity has been obtained for analyte Refractive Index 1.38. The maximum Amplitude sensitivity at Refractive index of 1.38 is $408 \mathrm{RIU}^{-1}$ and this is obtained at $710 \mathrm{~nm}$ wavelength.

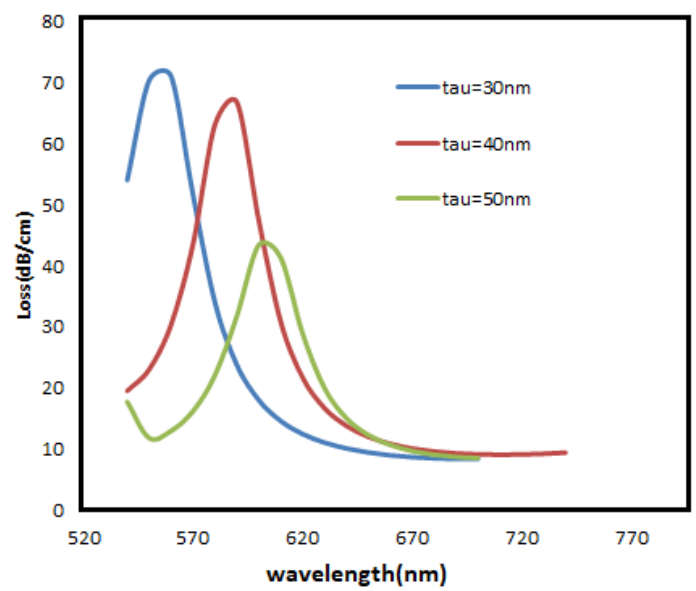

Fig. 4. loss curve for different gold thickness $\mathrm{t}_{\mathrm{au}}=30 \mathrm{~nm}, 40 \mathrm{~nm}, 50 \mathrm{~nm}$ and the parameters of PCF are include $\Lambda=2 \mu \mathrm{m}, \mathrm{d}_{1}=0.25 \times \Lambda, \mathrm{d}_{2}=0.5 \times \Lambda, \mathrm{d}_{\mathrm{c}}=0.3 \times \Lambda$ and $\mathrm{t}_{\mathrm{au}}=30 \mathrm{~nm}$.

Amplitude sensitivity and loss depth changes when the there is a change in the gold thickness. So these two parameters depend on the plasmonic material thickness. The loss curve for different plasmonic thickness at analyte $\mathrm{RI}=1.38$ is shown in Fig.4.The decrease in the loss depth is due to the damping effect of the gold [10]. Therefore, less energy from fundamental core mode is transferred to the plasmonic mode when compared with gold thickness of the lower value. There is also a shift in the curve when the thickness of plasmonic material ( $\mathrm{t}$ au)is increased. When the gold thickness tau is $30 \mathrm{~nm}$ the highest Amplitude Sensitivity which was observed was $382.3 \mathrm{RIU}^{-1}$ but when the thickness of plasmonic material was increased to $40 \mathrm{~nm}$ then the Amplitude sensitivity increased to 408.468 . When the gold thickness was increased to $50 \mathrm{~nm}$ peak loss became comparativelyless and amplitude sensitivity got reduced, so a constant thickness of gold $40 \mathrm{~nm}$ was used throughout the simulation. The highest Amplitude Sensitivity recorded for our design is 408.469 RIU-1 at a wavelength of 710nm.

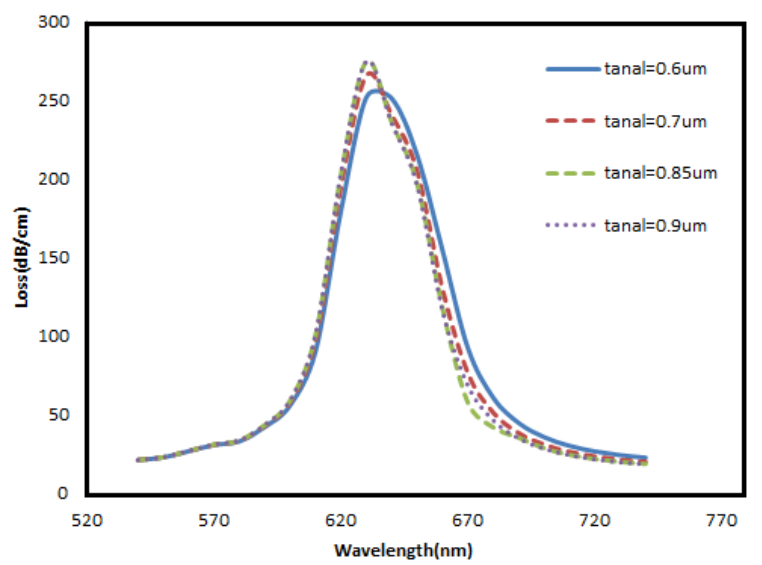

Fig. 5. Loss curve of fundamental mode for different analyte thickness $\mathrm{t}_{\text {anal }}=0.6 \mu \mathrm{m}, 0.7 \mu \mathrm{m}, 0.85 \mu \mathrm{m}, 0.9 \mu \mathrm{m}$. and the parameters of PCF are include $\Delta=2 \mu \mathrm{m}, \mathrm{d}_{1}=0.25 \times \Delta$, $\mathrm{d}_{2}=0.5 \times \Delta, \mathrm{d}_{\mathrm{c}}=0.3 \times \Delta$ and $\mathrm{t}_{\mathrm{au}}=30 \mathrm{~nm}$.

From the Fig.5 it can be understood that there is not much change in the loss depth when the thickness of the analyte is changed. The loss depth for the different analyte thickness of about $0.6 \mathrm{~nm}, \quad 0.7 \mathrm{~nm}, \quad 0.85 \mathrm{~nm}$ is $251.041(\mathrm{~dB} / \mathrm{cm})$, $265.614(\mathrm{~dB} / \mathrm{cm}), 274.584(\mathrm{~dB} / \mathrm{cm})$. Since there is not much difference in the loss depth as you can see in fig so constant thickness of analyte layer $t_{\text {anal }}=0.8 \mu \mathrm{m}$ has been assumed throughout the simulation.

The different analyte RI and its following resonant wavelength graph for the different tau thickness has been plotted in Fig.6. The regression analysis has been performed for different variation in gold thickness. The regression equation for gold thickness $t_{a u}=30 \mathrm{~nm}$ is given by 1431-4X-1350.1 and its corresponding R-Square values is 0.9673 and the regression equation of linear line fitting for gold thickness $t_{a u}=40 \mathrm{~nm}$ is $\mathrm{Y}=2389.3 \mathrm{x}-2599.9$ and its corresponding R-Square value is 0.9695 .

The $R^{2}$ value is almost nearer to unity, which indicates that the proposed sensor is linear ranging from 1.33 to 1.39. Since $R^{2}$ value is high and it is close to unity, this sensor which has been proposed by us can be implemented in practical sensing applications.

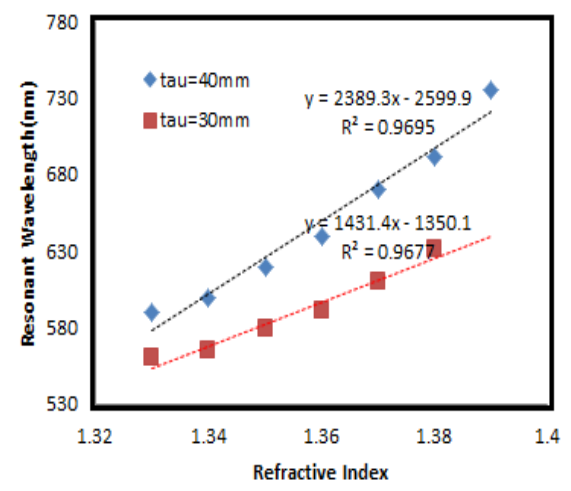

Fig. 6. Refractive index vs resonance wavelength graph for different gold layer thickness. 


\section{Authors Profile}

\section{CONCLUSION}

Through this paper we represent the design of PCF biosensor built on the phenomenon of SPR. The plasmonic coating covers the outer layer of the Photonic Crystal fiber to ease the fabrication process, we choose Gold. The sensitivities of $4300 \mathrm{~nm} / \mathrm{RIU}$ and $408.468 \mathrm{RIU}^{-1}$ can be accomplished by using the methodologies of wavelength and amplitude sensitivities. Since $R^{2}$ value is close to unity the sensor can be used in real time sensing applications. The proposed design could be used for biological detection and it has a wide sensing range from 1.33 to 1.39 .

\section{REFERENCES}

1. Shaimaa I Azzam, Mohamed Farhat O Hameed, Rania Eid Shehata, AM Heikal, and Salah SA Obayya. Multichannel photonic crystal fiber surface plasmon resonance based sensor. Optical and Quantum Electronics, 48(2):142, 2016.

2. MS Aruna Gandhi, S Sivabalan, P Ramesh Babu, and K Senthilnathan. Designing a biosensor using a photonic quasi-crystal fiber. IEEE Sensors Journal, 16(8):2425-2430, 2016.

3. Md Hasan, SanjidaAkter, Ahmmed Rifat, Sohel Rana, and Sharafat Ali. A highly sensitive gold-coated photonic crystal fiber biosensor Multidisciplinary Digital Publishing Institute, 2017. based on surface plasmon resonance. In Photonics, volume 4, page 18

4. Itaru Ishida, Tsuyoshi Akamatsu, Zhaoyang Wang, Yusuke Sasaki,Katsuhiro Takenaga, and Shoichiro Matsuo. Possibility of stack and draw process as fabrication technology for multi-core fiber. In 2013 Optical Fiber Communication Conference and Exposition and the National Fiber Optic Engineers Conference (OFC/NFOEC), pages 1-3.IEEE, 2013.

5. Duanming Li, Wei Zhang, Huan Liu, Jiangfei $\mathrm{Hu}$, and GuiyaoZhou.High sensitivity refractive index sensor based on multicoating photonic crystal fiber with surface plasmon resonance at near-infrared wavelength. IEEE Photonics Journal, 9(2):1-8, 2017.

6. Chao Liu, WeiquanSu, Qiang Liu, Xili Lu, Famei Wang, Tao Sun,and

7. Paul K Chu. Symmetrical dual d-shape photonic crystal fibers for surface

8. plasmon resonance sensing. Optics express, 26(7):9039-9049,2018.

9. Min Liu, Xu Yang, Ping Shum, and Hongtao Yuan. High-sensitivity birefringent and single-layer coating photonic crystal fiber biosensor based on surface plasmon resonance. Applied optics, 57(8):1883-1886.

10. Min Liu, Xu Yang, Bingyue Zhao, Jingyun Hou, and Ping Shum Square array photonic crystal fiber-based surface plasmon resonance refractive index sensor. Modern Physics Letters B, 31(36):1750352, 2017

11. AA Rifat, GhafourAmouzadMahdiraji, YM Sua, YG Shee, Rajib Ahmed, Desmond M Chow, and FR MahamdAdikan. Surface plasmon resonance photonic crystal fiber biosensor: a practica sensing.approach.IEEE Photonics Technology Letters, 27(15):1628-1631, 2015.

12. AhmmadRifat,GMahdirajiChow,YuShee,Rajib Ahmed and Faisal Adikan.Photonic Crystal Fiber based surface plasmon resonance sensor with selective analyte channels and graphene silver deposited core,Sensors, 15(5):11499-11510,2015.

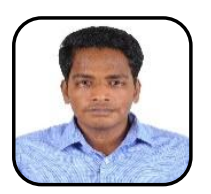

P Vinod Kumarcurrently in final year pursuing Engineering in Electronics and Communication at SRM Institute of Science and Technology, Kattankulathur, Kancheepuram, Tamil Nadu.

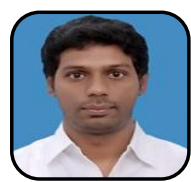

S Nivash currently working as a Assistant Professor in School of ECE, SRM Institute of science and technology,, Kattankulathur, Kancheepuram,

Tamil Nadu.

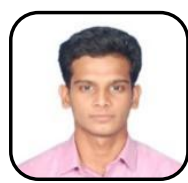

Mohammed Fahimullah Acurrently in final year pursuing Engineering in Electronics and Communication at SRM Institute of Science and Technology, Kattankulathur, Kancheepuram, Tamil Nadu.

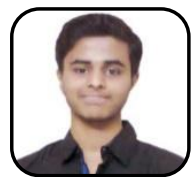

K Vinay Gokulcurrently in final year pursuing Engineering in Electronics and Communication at SRM Institute of Science and Technology, Kattankulathur, Kancheepuram, Tamil Nadu. 